INPLASY

PROTOCOL

To cite: Qin et al. Accuracy of lung ultrasound for the prediction of bronchopulmonary dysplasia in the newborn: a metaanalysis. Inplasy protocol 2021110075. doi: 10.37766/inplasy2021.11.0075

Received: 21 November 2021

Published: 21 November 2021

Corresponding author:

Qin Xiaojiao

qinxiaojiao2011@163.com

Author Affiliation:

Children's Hospital of Fudan

University.

Support: None.

Review Stage at time of this submission: Preliminary searches.

Conflicts of interest:

None declared.

\section{Accuracy of lung ultrasound for the prediction of bronchopulmonary dysplasia in the newborn: a meta- analysis}

Qin, XJ1; Luo, NX2; Sun, YH³; Zhang, XB

Review question / Objective: In the newborn, what is the diagnostic accuracy of lung ultrasound for the prediction of BPD? Participants: Neonate; Intervention:Lung ultrasound; Comparision: Clinical diagnosis; Outcome: Bronchopulmonary dysplasia; Study design: Cohort study.

Condition being studied: Bronchopulmonary dysplasia(BPD) is the most common sequelae of prematurity. With the development of neonatal treatment technology, the incidence of BPD is gradually increasing. BPD still causes significant mortality and has a negative impact on lung function and quality of life, which extends to adult life.Therefore, early diagnosis of BPD is very important. Lung ultrasound may be an early diagnostic tool for the prediction of Bronchopulmonary Dysplasia. It's a widely available, inexpensive, radiation-free and useful imaging modality to assess neonatal respiratory disease.

INPLASY registration number: This protocol was registered with the International Platform of Registered Systematic Review and Meta-Analysis Protocols (INPLASY) on 21 November 2021 and was last updated on 21 November 2021 (registration number INPLASY2021110075).

\title{
INTRODUCTION
}

Review question / Objective: In the newborn, what is the diagnostic accuracy of lung ultrasound for the prediction of BPD? Participants: Neonate; Intervention: Lung ultrasound; Comparision: Clinical diagnosis; Outcome: Bronchopulmonary dysplasia; Study design: Cohort study.
Condition being studied: Bronchopulmonary dysplasia(BPD) is the most common sequelae of prematurity. With the development of neonatal treatment technology, the incidence of BPD is gradually increasing. BPD still causes significant mortality and has a negative impact on lung function and quality of life, 
which extends to adult life.Therefore, early diagnosis of BPD is very important. Lung ultrasound may be an early diagnostic tool for the prediction of Bronchopulmonary Dysplasia. It's a widely available, inexpensive, radiation-free and useful imaging modality to assess neonatal respiratory disease.

\section{METHODS}

Search strategy: PubMed, Medline, the Cochrane Library, and Embase were searched for the relevant literature without any restriction in terms of language and time until October 31, 2021. The Medical Subject Headings (MeSH) terms and individual key terms such as "Ultrasound," "Ultrasonic," "ultrasonography," "Lung Ultrasound," "Lung Ultrasonic,"“Lung ultrasonography," "Bronchopulmonary dysplasia,""chronic lung disease,"“BPD," "Neonate," "Premature," "preterm infants," "Newborn,"and "Neonatal" were used for searching the databases.

Participant or population: All newborns, including term infants and premature infants.

Intervention: Lung ultrasound was performed within 28 days after birth.

Comparator: No comparator or control group; BPD is diagnosed according to the clinical definitions. Therefore, the clinical follow-up either at $\mathbf{2 8}$ days of life or at $\mathbf{3 6}$ weeks of gestation must be made.

Study designs to be included: Cohort studies, both prospective and retrospective.

Eligibility criteria: 1, Single or multiple regular lung ultrasound examinations were performed within 28 days after birth; 2, The clinical follow-up either at 28 days of life or at $\mathbf{3 6}$ weeks of gestation was made.

Information sources: PubMed, Medline, the Cochrane Library, and Embase were searched for the relevant literature without any restriction in terms of language and time until October 31, 2021. The Medical Subject Headings (MeSH) terms and individual key terms such as "Ultrasound," "Ultrasonic," "ultrasonography," "Lung Ultrasound," "Lung Ultrasonic,"“Lung ultrasonography," "Bronchopulmonary dysplasia," "BPD," "Neonate," "Premature,""preterm infants," "Newborn," and "Neonatal" were used for searching the databases.

Main outcome(s): Diagnostic accuracy of lung ultrasond for the prediction of BPD.

Additional outcome(s): Severity of BPD.

Quality assessment / Risk of bias analysis: The quality assessment was independently conducted and crosschecked by two authors with the Quality Assessment of Diagnostic Accuracy Studies 2 (QUADAS-2).If the conclusions are inconinsistent, the final decision will be made by the third-party experts or discussion.

Strategy of data synthesis: We will collect the information on true positive, false negative, false positive and true negative rate from each study. Data synthesis will be performed and results will be reported as summary estimate of sensitivity, specificity and DOR.

Subgroup analysis: Subgroup analysis will be performed based on gestational age, the timing of lung ultrasound, and BPD severity.

Sensitivity analysis: STATA 14.0 was selected for sensitivity analysis of the data in this study. The low quality studies that had a large impact on sensitivity was excluded to evaluate the robustness of the model.

Language: No restrictions on language.

Country(ies) involved: China.

Keywords: Lung u Itrasoud, Bronchopulmonary Dysplasia, Neonate. 
Contributions of each author:

Author 1 - Qin Xiaojiao.

Author 2 - Luo Ningxin.

Author 3 - Sun yinghua.

Author 4 - Zhang xiaobo. 